\title{
Electromyographic Pattern of Respiratory Muscles in Young People during Incremental Exercise
}

\author{
Priscilla Anjos de Sousa ${ }^{1}$, Luciana Dias Chiavegato ${ }^{1}$, Wellington B Vieira ${ }^{1}$, Rubens Alexandre da Silva ${ }^{2}$, \\ Maryanne Martins Gomes de Carvalho ${ }^{1}$, Paulo Rui de Oliveira ${ }^{1}$, Bruno Mazziotti ${ }^{1,6,7}$, Tamara de Paula e \\ Mancilha $^{3}$, Runer Augusto Marson ${ }^{3,4,5}$, César Ferreira Amorim ${ }^{1,5,6^{*}}$ \\ ${ }^{1}$ Master and Doctoral Program in Physical Therapy-University of City of São Paulo -UNICID, Tatuapé - SP, Brazil \\ ${ }^{2}$ Center for Health Science Research, Laboratory of Functional Evaluation and Human Motor Performance, Doctoral and Master Rehabilitation \\ Program UEL/UNOPAR, Londrina-PR, Brazil. \\ ${ }^{3}$ Postgraduation in Exercise Physiology, Department of Physiological Sciences, Federal University of São Carlos, UFSCar \\ ${ }^{4}$ Brazilian Army Research Institute of Physical Fitness, IPCFEx, Rio de Janeiro -RJ, Brazil \\ ${ }^{5}$ Post Doctoral Program, Postgraduate Program in Biomedical Engineering, University of Vale do Paraiba (UNIVAP), Sao Jose dos Campos-SP, Brazil. \\ ${ }^{6}$ Sport Club Corinthians Paulista, Lab Corinthians R9, Tatuapé - SP, Brazil \\ ${ }^{7}$ Shandong Luneng Taishan Football Club, Jinan, Shandong, China.
}

Received: June 7, 2016; Accepted: August 9, 2016; Published: September 19, 2016

*Corresponding author: César Ferreira Amorim, PhD, Professor, Master and Doctoral Program in Physical Therapy-University of São Paulo City-UNICID, Tatuapé - SP, Brazil. Postgraduate Program in Biomedical Engineering, Universidade do Vale do Paraiba (UNIVAP), Sao Jose dos Campos-SP, Brazil. Tel: +55 12 39224069, Fax: +55 12 39424736; Email Address: cesar@emgsystem.com.br

\begin{abstract}
During exercise, the muscles involved inbreathing is progressively recruited due to increased demand in respiratory effort. Surface electromyography (sEMG) is a technique that allows evaluation of these muscles providing information on the recruitment of motor units. The aim of this study was describe the electrical activity of the upper trapezius, sternocleidomastoid and intercostal sternum muscles in active and sedentary young subjects during incremental exercise test. Cross-sectional research design, including 20 young adults of both genders, aged 18to 36 years participated of study. The EMG signal amplitudes were analyzed in the time domain from muscular activity of the upper trapezius, sternocleidomastoid and intercostal sternum muscles, in relation to the variation of the load imposed on the incremental exercise. A mixed linear adjustments model was used to study the effects of the load, groups (active and sedentary) and the interaction (load x group) .The results there was no significant difference between-groups and interaction effects (group $\mathrm{x}$ load). Significant increase in the EMG signal amplitude was however found during the increase of the load for the three muscles studied. There was an increase in the amplitude of the electromyographic signal of accessories respiratory muscles in relation to the load increase during incremental exercise test.
\end{abstract}

Keywords: Physical Activity; Respiratory Muscles; Electromyography; Ergometry

\section{Introduction}

The performance of exercise depends on the functional state of the respiratory, cardiovascular [1] and musculoskeletal systems [2]. Cardiorespiratory fitness is related to the performance capacity of the large dynamic muscles in moderate intensity exercise for prolonged periods [1].
Ventilation occurs through the coordinated contraction of specific muscle groups, which in inspiration produces enough energy to distend the pulmonary structures $[3,5]$. In the healthy individual the ventilatory requirements during exercise, are easily achieved because the breathing muscles are anatomically suited for increasing ventilatory requirements and neural regulation of respiration is ideal [6].

It should be emphasized that the accessory respiratory muscles are progressively recruited with the increased ventilatory demand. Thus, sharing the load required to bear hyperpnea, shallow and rapid breathing that occurs during exercise [6,7].

Among the respiratory muscles recruited during exercise, the external intercostal sternum sternums are considered important because of their important postural and respiratory role in stabilizing and maintaining the shape and integrity of the rib cage when the ribs elevate and expand the chest during inspiration. The sternocleidomastoid is also considered by some authors the most important accessory muscle of inspiration along with the upper trapezius whose ventilatory role is to assist in the inspiration on effort helping to raise the rib cage [8].

Understanding the physiological changes of the respiratory muscles before physical effort is a largely explored subject in exercise physiology. The proper functioning of these muscles results in an improvement of the performance of elite athletes, besides its great importance for chronic lung diseases, Parkinson's, and cardiovascular disorders whose clinical changes result in weakness of these muscles [9]. 
Surface electromyography ( $\mathrm{sEMG}$ ) has been widely used as an aid in clinical diagnosis for over 40 years [10]. The use of sEMG makes it possible to evaluate the muscle activity in the temporal domain of the amplitude of each muscle in the body separately or together to complete the movement. Becoming a valuable tool in teaching and learning processes such as self-regulation of contraction through biofeedback, in deep relaxation process and muscle stress management $[10,11,12]$.

The sEMG collection system consists of electrodes, amplifiers, filters and recording devices. These electrodes convert the electrical signal resulting from the depolarization process of the muscle fibers, into an electrical signal that can be processed in an amplifier used for nerve conduction tests and kinesiological investigations [13]. Root Mean Square (RMS ) is the most used analytical method for analyzing the time behavior of the EMG signal. RMS amplitude is directly related to the recruitment of motor units $[9,10,14,15]$. Thus the importance of this study, which through a previously well documented tested and validated by an instrumented biomechanical evaluation, aims to address the relationship between the accessory respiratory muscles and incremental exercise loads in young subjects who engage in regular physical activity, or who are sedentary, in order to recognize and describe the electrical activity of the upper trapezius, sternocleidomastoid and intercostal sternums muscles.

\section{Methods}

A cross-sectional observational research design from the Motion Analysis Laboratory at the University Cidade de São Paulo study was conducted. The project was approved by the Ethics Committee (protocol number 13657056 - UNICID-SP) and participants signed an informed consent.

\section{Subject}

The study was performed with healthy, college adults, of both genders, aged between 18-36 years; individuals with any diagnosed respiratory disease, respiratory symptoms, and chest deformities, pregnant, musculoskeletal, cognitive or cardiovascular anomalies were excluded.

The variables analyzed were the SEMG signal amplitudes in time domain of the upper trapezius, sternocleidomastoid and intercostal sternum muscles variation in relation to the load variation imposed on the incremental exercise of the lower limbs.

\section{Procedures}

All subjects underwent physical examination to measure vital signs: heart rate, respiratory rate, oxygen saturation and blood pressure. The subjects involved in the study answered the Physical Activity Readiness Questionnaire (Par- Q) which aims to assess readiness for physical activity [16]. If even one of the seven questions, got a positive answer the subject required clinical evaluation and medical clearance to participate in the study. If for some reason the person did not get a bill of health he/ she would be excluded from the study in order to avoid any risks.

Then, the subjects answered the Baecke Questionnaire of habitual physical activity [17,19], which consists of 16 questions about occupational physical activity, leisure exercise and physical activity in leisure and locomotion. The purpose of this questionnaire was to classify the individual as physically active or sedentary. In addition, we adopted the concept established by the American College of Sports Medicine (ACSM) [20] of sedentary lifestyle, defined as non-participation in at least 30 minutes of moderate-intensity physical activity at least 3 days per week, for at least 3 months. The subjects were then divided into two groups, active and sedentary. To be included in the study groups individuals should be rated by the two methods and the results should be the same.

\section{Placement of surface electrodes}

To collect the SEMG signal of the upper trapezius, sternocleidomastoid and intercostal sternum muscles, disposable circular adhesive silver / silver chloride (Ag / $\mathrm{AgCl}$ ) surface electrodes, with a $10 \mathrm{~mm}$ diameter (Medi Trace TM / Kendall), an inter-electrode distance of $20 \mathrm{~mm}$ center to center were used. As reference electrode, a rectangular metal electrode was used fixed to the right wrist of volunteers would cancel the interference effect mechanism of external electrical noise [21].

To determine the location of the electrodes, anatomical bony prominences, muscle architecture and physiological cross-sectional area, a region of higher concentration of motor endplates, were used as reference in the assessment procedure. Markings for electrode placement were made with skin pencil through anthropometry with tape, after previous site cleaning with cotton and alcohol to reduce skin impedance. All the recommendations were followed as suggested by DE LUCA: The Use of Surface Electromyography in Biomechanics (1997) $[22,24]$.

Due to the location of the evaluated muscles, to reduce the interference of the cardiac signal, the evaluation was only on the right side of the subject.

\section{Capture and analyses of electromyographic signal}

The electromyography system used was the EMG System of Brazil model EMG800C USB communication, powered by internal battery, immune to main electrical interference, comprising analog to digital converter with 16-bit resolution, signal conditioner integrated with amplification gain of 2000 times, filter $20-500 \mathrm{~Hz}$ Butterworth bandpass and bipolar electrodes with pre-amplification EMG channels $[10,21]$.

For the strength, speed and pedal cadence sensor channels a band pass 0-100 Hz Butterworth filter was selected. An acquisition program and signal processing EMGLab V1.1 EMG System of Brazil brand 2010 version software with sampling frequency by $2 \mathrm{kHz}$ channel collection time of 24 minutes maximum was used [21].

The EMG system was coupled to the computer. This allowed, during the incremental exercise, the trace related to the cycle ergometer load to be followed so that the load increments could be performed with precision. Analysis in continuous screens was 
initially used for evaluation of Maximum Voluntary Contraction (MVC) with the manovacuometer, and then throughout the incremental exercise on the lower limb cycle ergometer.

Data recorded by the instruments were analyzed by EMGLab (EMG System, Brazil) software that provides all the information related to signal amplitude including RMS average. The data, recorded simultaneously, were analyzed by quantitative method in the dominion amplitude. The values of electrical muscle activity ( $\mu$ V RMS) were normalized individually by MVC.

The RMS averages of the muscles were obtained in sliding windows every $200 \mathrm{~m}$. This procedure was applied with the initial load, $50 \%$ load and $100 \%$ of the total load carried by the individual in the assessment procedure.

\section{MVC Evaluation}

After instrumentation and preparation of the individual, evaluation was performed with analog manometer (Instrumentation Industries Ltda.). Associated with the assessment of Maximum Inspiratory Pressure (MIP), electromyographic analysis of the upper trapezius sternocleidomastoid and intercostal sternum muscles aiming to get an MVC that could be used as standard electromyographic signal. The MIP, measured in $\mathrm{cm} \mathrm{H}_{2} \mathrm{O}$, was obtained with the subject seated. The procedure was performed 3 times with a lapse of 1 minute between each measurement. The best value among the reviews was adopted.

The RMS averages of the muscles were obtained in sliding windows every $200 \mathrm{~m}$. This was accomplished with the initial load, $50 \%$ load and $100 \%$ of the total load carried out by the individual in the assessment procedure.

The MIP, measured in $\mathrm{cm}_{2} \mathrm{O}$ was measured with the subject seated. The procedure was performed 3 times with a lapse of 1 minute between each turn and the best value among the reviews was adopted.

\section{Incremental Exercise}

The choice of the Billat protocol (2001) proved to be the most appropriate in relation to other incremental protocols because it accomplishes the increments of $25 \mathrm{~W}$ in a load cycle ergometer every 3 minutes. It also keeps a constant speed average of 22.5 $\mathrm{km} / \mathrm{h}$ until subjective fatigue is reported by the individual being evaluated [25].

Considering that the Billat protocol is performed until subjective fatigue, to rate the perceived exertion 30 seconds before increasing the load of the lower limbs cycle ergometer, subjects answered the Borg Scale [26,27]. Along with the Borg Scale, peripheral oxygen saturation and heart rate were also measured.

To ensure the integrity of the participants, symptoms for stopping the test as established by the ACSM (2007) [20] were adopted. After the incremental protocol, vital signs were measured and recorded again.

\section{Statistical analysis}

In the descriptive statistical analysis, continuous variables were presented as mean and standard deviation, and categorical variables were presented as contingency proportion and percentage table.

The Shapiro-Wilk test was used to test the normality of the data distribution of the studied variables. Among the findings, variables with parametric distribution, described as mean and standard deviation and nonparametric variables which were presented as median and interquartile range were found.

The model for linear adjustments with mixed structure of variance and covariance to study the effect of load (physically active and sedentary) group effect and load x group interaction on the variables studied was used. All analyzes were performed with SPSS software version 13.0 for Windows (Chicago, IL, USA) and the significance level was set at $5 \%$. Sample calculation.

\section{Results and Discussion}

There were twenty-four participants from which four were excluded, one for not being able to perform physical exercise accordingly to the Par- $Q$ questionnaire; the other three were rejected due to a discrepancy between the level of physical activity of the Baecke Questionnaire of habitual physical activity and the ACSM concept. The 20 subjects were divided into two equal study groups, active or sedentary. Subjects were paired by age and BMI. The characteristics and demographic variables of the subjects are described in Table 1.

Among the vital signs measured before and after incremental exercise, the end-systolic pressure was the only variable that was different between the groups with 132 (7.89) mm Hg for the active group and 120 (14:14) mmHg for the sedentary group with a value Po $\mathrm{f} .03$. As for initial and end test values: heart and respiratory rates for both groups and systolic blood pressure for the physically active group also proved to be statistically different (Table 2).

Table 1: Characteristics and demographic variables of the 20 study participants.

\begin{tabular}{|l|l|l|}
\hline Variable & Actives & Sedentary \\
\hline $\begin{array}{l}\text { Gender (Female/ } \\
\text { Male) }\end{array}$ & $5 / 5$ & $5 / 5$ \\
\hline Age (years) & $25.70(5.29)$ & $23.10(4.23)$ \\
\hline Height (meters) & $1.73(0.1)$ & $1.70(0.09)$ \\
\hline $\begin{array}{l}\text { Weight } \\
\text { (kilograms) }\end{array}$ & $74.75(13.03)$ & $75.78(21.77)$ \\
\hline $\begin{array}{l}\text { Body Mass Index } \\
\text { (BMI) }\end{array}$ & $25.08(3.65)$ & $25.99(6.63)$ \\
\hline Smoker (Yes/No) & $1 / 9$ & $1 / 9$ \\
\hline Par-Q (Fit/Unfit) & $10 / 0$ & $10 / 0$ \\
\hline Baecke* & $9.90(0.71)$ & $7.37(0.81)$ \\
\hline
\end{tabular}

Continuous variables are expressed as mean and standard deviation (SD), BMI (body mass index); Par-Q (Physical Activity Readiness Questionnaire); Baecke (Baecke questionnaire of habitual physical activity).

* Value for cutting Baecke 9. 
With respect to the values obtained by the Borg scale during incremental exercise loads the values ranged from 0 to 10 . The values under each load and for each group, as well as the $P$ value for group effect are shown in Table 3. There was no statistically significant difference between groups.

The total test time was 14 [5] minutes for the physically active and 11[5] minutes for the sedentary group. There was no statistically significant difference between the total testing time of the two the groups, the value P was .22. The largest testing time of 20 minutes was from a subject of the physically active group. In Table 4 the RMS averages of the upper trapezius, sternocleidomastoid and intercostal sternum muscles of the participants during the evaluation of the MVC and during the loads of incremental exercise.

Figure 1 shows the behavior of the upper trapezius, sternocleidomastoid and intercostal sternum muscles for the

Table 2 : Clinical signals pre and post incremental test.

\begin{tabular}{|c|c|c|}
\hline Variables & Active & Sedentary \\
\hline Initial HR (bpm) & $76.80(7.35)$ & $75.00(11.42)$ \\
\hline $\begin{array}{l}\text { Final HR (bpm) } \\
\text { Value p pré and post } \\
\text { exercise }\end{array}$ & $\begin{array}{l}143.30(20.97) \\
<.001\end{array}$ & $\begin{array}{l}137.60(20.94) \\
<.001\end{array}$ \\
\hline $\begin{array}{l}\text { Initial RF (breaths/ } \\
\text { min) }\end{array}$ & $16.30(2.95)$ & $17.30(2.75)$ \\
\hline $\begin{array}{l}\text { Final RF (breaths/ } \\
\text { min) } \\
\text { Value p pré and post } \\
\text { exercise }\end{array}$ & $\begin{array}{l}23.30(3.59) \\
<.001\end{array}$ & $\begin{array}{l}24.80(3.68) \\
<.001\end{array}$ \\
\hline Initial $\mathrm{SpO}_{2}(\%)$ & $96.40(0.70)$ & $96.50(1.08)$ \\
\hline $\begin{array}{l}\text { Final } \mathbf{S p 0}_{2}(\%) \\
\text { Value p pré and post } \\
\text { exercise }\end{array}$ & $\begin{array}{l}96.20(0.79) \\
.44\end{array}$ & $\begin{array}{l}96.40(1.17) \\
.78\end{array}$ \\
\hline $\begin{array}{l}\text { Initial systolic } \\
\text { pressure (mmHg) }\end{array}$ & $119(8.76)$ & 116 (6.99) \\
\hline $\begin{array}{l}\text { Final systolic } \\
\text { pressure (mmHg) } \\
\text { Value p pré and post } \\
\text { exercise }\end{array}$ & $\begin{array}{l}132(7.89) \\
<.001\end{array}$ & $\begin{array}{l}120(14.14) \\
.30\end{array}$ \\
\hline $\begin{array}{l}\text { Initial diastolic } \\
\text { pressure (mmHg) }\end{array}$ & $76(5.16)$ & 75 (7.07) \\
\hline $\begin{array}{l}\text { Final diastolic } \\
\text { pressure (mmHg) } \\
\text { Value p pré and post } \\
\text { exercise }\end{array}$ & $\begin{array}{l}74(8.43) \\
.44\end{array}$ & $\begin{array}{l}71(9.94) \\
.16\end{array}$ \\
\hline \multicolumn{3}{|c|}{$\begin{array}{l}\text { Continuous variables are expressed as mean and standard deviatior } \\
\text { (SD), HR (Heart Rate); bpm (beats per minute), RF (Respiratory } \\
\text { Frequency), SpO2 (Oxygen Saturation); mmHg (millimeters of } \\
\text { mercury). }\end{array}$} \\
\hline
\end{tabular}

Table 3 : Borg scale during incremental exercise.

\begin{tabular}{|l|l|l|l|}
\hline Variables & Active & Sedentary & P value \\
\hline Initial load & $1.10(1.33)$ & $1.60(1.41)$ & .42 \\
\hline $\mathbf{5 0 \%}$ load & $3.13(1.46)$ & $2.79(1.47)$ & .66 \\
\hline $\mathbf{1 0 0 \%}$ load & $6.50(2.51)$ & $5.70(1.77)$ & .42 \\
\hline
\end{tabular}

Continuous variables are expressed in mean and standard deviation (SD) $. P<0.05$.

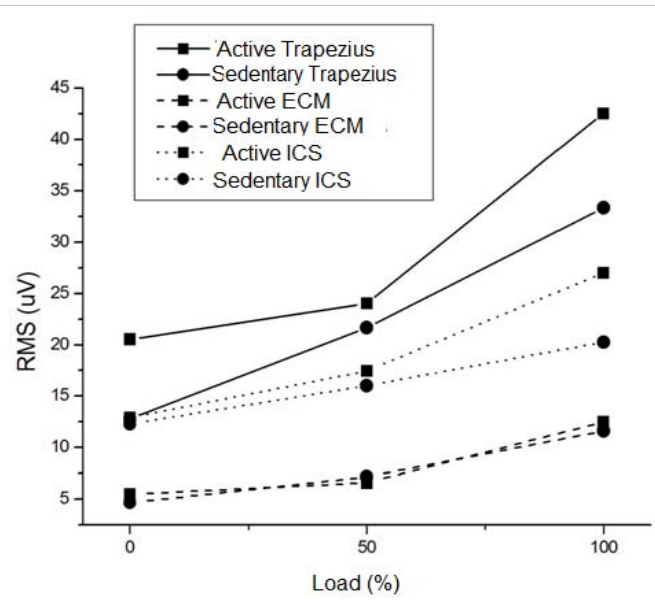

Figure 1: RMS average of muscles during loads of incremental exercise.

two study groups (physically active and sedentary) during incremental exercise loads.

Pvalues for group effect and group x load were not statistically significant. Regarding the load effect for the three muscles tested the value $P$ was statistically significant: .002 for the upper trapezius muscle $<.001$ for the sternocleidomastoid muscle and .013 for the external intercostal sternum muscle.

The analysis of the behavior of the electromyographic signal accessory respiratory muscles in young active and sedentary people, in loads of different incremental exercise on a lower limb cycle ergometer demonstrated that its amplitude increases following the increase of the load during the test for both groups. For the three studied muscles, the upper trapezius, sternocleidomastoid and intercostal sternum, there was a linear relationship of increased activation with increase of load. Andrade et al (2005) had already established that physical exercise increases the performance of inspiratory muscles thereby increasing the tolerance to activities [28]. Noble et al (2006) observed an increase in electromyographic activity of the respiratory muscles during progressive loading of respiratory effort [29].

By observing the RMS average of the muscles evaluated during incremental exercise and comparing it with the values obtained at the maximum voluntary contraction, we observed that most participants stopped the test before even reaching the maximum stress tolerated by the cardiorespiratory system claiming fatigue of leg muscles since it is an exercise that requires a lot from the lower limb muscles. This finding corroborates the ACSM guidelines (2007) which state that this method of exercise is regarded as highly dependent on the routine activities of the participants, their fitness, strength in the lower limbs and motivation of the individual being evaluated [20].

This type of behavior is also mentioned by Neder et al (2002), with the sensations of muscular discomfort, pain and fatigue commonly referred to as the major "symptoms" limiting the continuation of the activity in normal untrained subjects. Indeed, this could be anticipated, considering the importance 
of cardiovascular/muscular in physiologically limiting the progression of activity. Still regarding the appearance of symptoms that limit the continuation of the incremental test Neder et al (2002) also advocate that the sensations of fatigue appear to have an onset threshold before rising exponentially. This effect was also observed in our study where the values obtained in the Borg scale were low in the initial load and at 50\% and increased substantially at $100 \%$ load [30].

Despite its important role as accessory inspiratory muscle among the muscles tested, the sternocleidomastoid muscle in our study showed the lowest activation during the incremental test. Contradicting the study by Ratnovsky et al (2005) which showed that it increases with high efforts and does nearly $10 \%$ of the inspiratory work [31].

Eastwood et al. (2001) investigated the effect of increased airway resistance in athletes and sedentary individuals and found that during exercise both groups maintained a low respiratory rate unlike our study in which individuals of both groups had a statistically significant increase in respiratory rate. Eastwood et al. (2001) also observed a decrease in oxygen saturation at peak load, which was not observed in our study since our rate of arterial oxygenation remained unchanged. And as in our study, Eastwood et al. (2001) demonstrated that the efficiency of the respiratory muscles was similar in both groups [32].

In clinical practice the results of this study can be used as an important tool for physical therapy and rehabilitation of musculoskeletal, biomechanical and functional disorders. Knowing that exercise with increasing loads on a lower limbs cycle ergometer increases the activation of the upper trapezius, sternocleidomastoid and intercostal sternum muscles this finding makes it possible to offer training programs of this musculature by professionals.

This study conducted a quality instrumentation in which the lower limbs cycle ergometer had a force transducer which's synchronized to the acquisition module and to the computer could make perfect adjustments to the load increment, besides controlling the speed and movement gradation of the lower limbs with the electrogoniometer.

During data collection it was possible to see that the lifestyle and the kind of physical activity performed by the participants limited their performance in the test. And that the location and depth of the muscles is possibly crosstalk, i.e., capture of electrical signal from adjacent muscles.

Future studies with a larger number of participants will allow for a better analysis of the behavior of accessory respiratory muscles, and may further the study of the behavior of other muscles such as the scalene pectoralis major.

Therefore, this study showed that the muscles assessed, the upper trapezius, sternocleidomastoid and intercostal sternum, increase in amplitude EMG signal, i.e., the electrical activity of the muscle, increases proportionally to the load of the clinical exercise test on a lower limbs cycle ergometer for both groups, active or sedentary.

\section{Acknowledgements}

Experimental data for this study were collected in the César F. Amorim Laboratory for Biomechanics and Human Movement and Rehabilitation at the University of City of São Paulo - UNICID. This work is partially supported by the CNPq under grant MCTI/ CNPQ/Universal 14/2014. Process:458880/2014-0.

\section{References}

1. García-Ortiz L, Grandes G, Sánchez-Pérez A, Montoya I, IglesiasValiente JA, Recio-Rodríguez JI, et al. Effect on Cardiovascular Risk of an Intervention by Family Physicians to Promote Physical Exercise Among Sedentary Individuals. Rev EspCardiol. 2010;63(11);12441252. doi:10.1016/S1885-5857(10)70249-8.

2. Morelli KM, Garcia JD, Brown L, Farthing AK, Miller JL, Warren GL. Clinical Measures Predicting Knee Extensor Muscle Activation During a Maximal Voluntary Isometric Contraction. J Exerc Sports Orthop. 2016;3(1);1-10.

3. Enright S, Chatham K, Ionescu AA, Unnithan VB, Shale DJ. Inspiratory Muscle Training Improves Lung Function and Exercise Capacity in Adults With Cystic Fibrosis. Chest. 2004;126(2);405-411. doi:10.1378/chest.126.2.405.

4. Shumacker PT, Leff AF. Fisiologia respiratória: fundamentos e aplicações. São Paulo: Interlivros. 1998.

5. Sheel AW, Derchak PA, Pegelow DF, Dempsey JA. Threshold effects of respiratory muscle work on limb vascular resistance. Am J Physiol Heart Circ Physiol. 2002;282(5);H1732-8. doi: 10.1152/ ajpheart.00798.2001.

6. Kendal FP, McCreary EK, Provance PG. Músculos, provas e funções. 4 ed. São Paulo: Editora Manole LTDA.; 2003.

7. Romer LM, Polkey MI. Exercise-induced respiratory muscle fatigue: implications for performance. J Appl Physiol. 2008;104(3);879-888. doi: 10.1152/japplphysiol.01157.2007.

8. Guyton AC, Hall JE. Tratado de fisiologia médica. 10 ed. Rio de Janeiro: Guanabara Koogan S.A. 2002.

9. Enright SJ, Unnithan VB. Effect of inspiratory muscle training intensities on pulmonary function and work capacity in people who are healthy: a randomized controlled trial. PhysTher. 2011;91(6);894-905. doi: 10.2522/ptj.20090413.

10. Marson RA. Relationships between surface electromyography and strength during isometric ramp contractions. In: Naik GR, editor. Computational Intelligence in Electromyography Analysis: A Perspective on Current Applications and Future Challenges. Rijeka: Intech; 2012;53-64. doi: dx.doi.org/10.5772/48819.

11. Klyvia JRM, Cunha RA, Lins OG, Cunha DA, Silva HJ. Eletromiografia de Superfície: Padronização da técnica. Neurobio. 2010;73(3).

12. Cohen BA. Biofeedback: Principles and Practice for Clinicians. 3 ed. Baltimore: Willians and Wilkins.; 1989.

13. Bhajan S, PanizzaJA, Finucane KE. Diaphragm electromyogram root mean square response to hypercapnia and its intersubject and dayto-day variation. JAppl Physiol. 2005;98;274-281. doi: 10.1152/ japplphysiol.01380.2003.

14. Silva AMO, Maturi S, Boin IFSF. Comparison of Surface Electromyography in Respiratory Muscles of Healthy and Liver Disease Patients: Preliminary Studies. Transplant Proc. 2011;43;1325-1326. doi: 10.1016/j.transproceed.2011.03.058. 
15. Silva AMO, Cliquet A, Boin IFSF. Profile of Respiratory Evaluation Through Surface Electromyography, Manovacuometry, and Espirometry in Candidates on the Liver Transplant Waiting List. Transplant Proc. 2012;44;2403-2405. doi: 10.1016/j. transproceed.2012.07.136.

16. Thomas S, Reading J, Shephard RJ. Revision of the Physical Activity Readiness Questionnaire (PAR-Q). Can J Sport Sci. 1992;17(4):338345.

17. Baecke JAH, Burema J, Frijters JER. A short questionnaire for the measurement of habitual physical activity in epidemiological studies. Am J Clin Nutr.1982; 936-942.

18. Florindo AA, Latorre MRDO. Validation and reliability of the Baecke questionnaire for the evaluation of habitual physical activity in adult men. Rev Bras Med Esporte. 2003;9(3);121-128. doi:dx.doi. org/10.1590/S1517-86922003000300002.

19. Florindo AA ,Latorre MRDO, Jaime PC, Tanaka T, Zerbini CAF. Metodologia para avaliação da atividade física habitual em homens com 50 anos ou mais. Rev Saúde Pública. 2004;38(2);307-314. doi:dx. doi.org/10.1590/S0034-89102004000200022.

20. American College of Sports Medicine. Diretrizes do ACSM para os Testes de Esforço e Sua Prescrição. 7 ed. Rio de Janeiro: Guanabara Koogan. 2007.

21. Amorim CF, Giannasi LC, Ferreira LM, Magini M, Oliveira CS, de Oliveira $\mathrm{LV}$, et al. Behavior analysis of electromyographic activity of the masseter muscle in sleep bruxers. J Body Mov Ther. 2010;14(3);234238. doi: 10.1016/j.jbmt.2008.12.002.

22. De Luca CJ. The Use of Surface Electromyography in Biomechanics. JAB. 1997;13(2);135-163.

23. Basmajian JV, De Luca CJ. Muscle alive: their function revealed by electromyography. 5 ed: Baltimore: Williams and Wilkins. 1985.
24. Criswell E. Cram's introduction to surface electromyography. 2ed: KindleEdition. 1998.

25. Azevedo, PHSM de, Garcia, A, Duarte, JMP, Rissato, GM, Carrara, VKP, Marson RA. Anaerobic threshold and bioenergetics: A Didactic Approach. J Phys Education. 2009; 20(3);453-464. doi: 10.4025/ reveducfis.v20i3.4743.

26. Chen MJ, Fan X, Moe ST. Criterion-related validity of the Borg ratings of perceived exertion scale in healthy individuals: a meta-analysis. JSports Sci. 2002;20(11);873-899. doi:10.1080/026404102320761787.

27. Robertson R, Goss F, Michael T, Moyna N, Gordon P, Visich P, et al. Validity of the Borg perceived exertion scale for use in semirecumbent ergometry during immersion in water. Percept Mot Skills. 1996;83(1);3-13. DOI:10.2466/pms.1996.83.1.3.

28. Andrade AD, Silva TNS, Vasconcelos H, Marcelino M, Machado MGR, Filho VCG et al. Inspiratory activation during threshold therapy in elderly healthy and patients with COPD. J Electromyogr Kinesiol. 2005;15(6);631-639. doi:10.1016/j.jelekin.2005.06.002.

29. Nobre MEP, Lopes F, Cordeiro L, Marinho PEM, Silva TNSS, Amorim $\mathrm{C}$ et al. Inspiratory muscle endurance testing: Pulmonary ventilation and electromyographic analysis. Respir Physiol Neurobiol. 2007;155(1);41-48. doi:10.1016/j.resp.2006.04.005

30. Neder JA, Nery LE. Teste de Exercício Cardiopulmonar. J Pneumol. $2002 ; 28(3)$

31. Ratnovsky A, Elad D. Anatomical model of the human trunk for analysis of respiratory muscles mechanics. Respir Physiol Neurobiol. 2005;148(3);245-262. doi:10.1016/j.resp.2004.12.016.

32. Eastwood PR, Hillman DR, Finucane KE. Inspiratory muscle performance in endurance athletes and sedentary subjects. Respirology. 2001;6;95-104. doi: 10.1046/j.1440-1843.2001.00314.x 